\title{
Poliposis adenomatosa familiar. Alternativas terapéuticas y estudio de los familiares
}

\author{
Gonzalo Soto D, Francisco López-Köstner, Alejandro Zárate C, \\ Fernando Vuletin S, Alejandro Rahmer O, Francisca León G, \\ Álvaro Zúníga $D$.

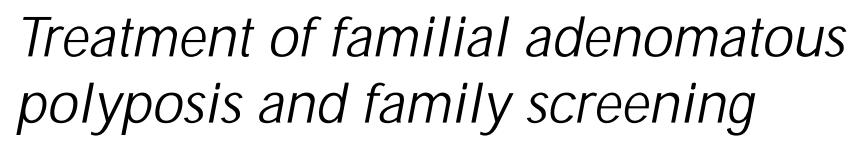

Background: To reduce the mortality associated to Familial Adenomatous Polyposis (FAP), screening of close relatives of patients with the disease is crucial. Aim: To analyze the results of the surgical treatment of patients with FAP, and to evaluate the family screening. Patients and Methods: Clinical records of patients operated in our institution since 1977, were reviewed analyzing surgical and pathological results, and follow up. In their family members, we evaluated and analyzed the performance of screening tests, former surgeries, history of disease-related cancer and mortality, all due to FAP. Results: Between January 1977 and August 2002, 15 patients were operated on. Of these, only $33 \%$ consulted on the setting of a familial screening. A proctocolectomy and terminal ileostomy was performed in $27 \%$ of patients; $20 \%$ had a proctocolectomy and ileal pouch, and $53 \%$ underwent a total colectomy with ileo-rectal anastomosis. Morbidity and mortality were $7 \%$ and $0 \%$, respectively. Twenty percent had a colorectal cancer. During a median of 68 months follow-up, the disease-related survival was 92\%; no cancer of the rectal stump was detected. Of the 122 family members identified, only 33\% with clear indication of screening underwent a colonoscopy. Twenty-nine percent had a confirmed FAP and were operated: in $61 \%$ of them a colorectal cancer was found, and 91\% of these died. Conclusions: The results of the surgical treatment of FAP are satisfactory. Nevertheless, family screening should be improved to reduce the high rates of mortality revealed in the study of other family members (Rev Méd Chile 2005; 133: 1043-50).

(Key Words: Adenomatous polyposis coli; Colorectal neoplasms; Familial adenomatous polyposis)

Recibido el 1 de diciembre, 2004. Aceptado el 4 de abril, 2005.

Trabajo parcialmente financiado por proyecto Fondecyt № 1040827.

Departamento de Cirugía Digestiva, Facultad de Medicina, Pontificia Universidad Católica de Chile.

L a poliposis adenomatosa familiar (PAF) y el cáncer de colon hereditario no poliposo (HNPCC) son enfermedades hereditarias predispo-

Correspondencia a: Dr. Francisco López K. Departamento de Cirugía Digestiva, Pontificia Universidad Católica de Chile. Marcoleta 367 Casilla 114-D, Santiago. Teléfono: 3543221-3543870. Fax: 6329620. E mail: flopez@med.puc.cl, cdigest@med.puc.cl nentes del desarrollo de cáncer colorrectal (CC) habitualmente antes de los 50 años ${ }^{1}$. La PAF se define clásicamente por la presencia de más de 100 pólipos adenomatosos en el colon y recto (Figura 1), y constituye una enfermedad hereditaria generada por una mutación en la línea germinal del gen APC (adenomatous polyposis coli) ${ }^{1-10}$. Si bien su expresión fenotípica más frecuente es la poliposis colorrectal, también puede afectar a otros parén- 
Figura 1. Múltiples pólipos adenomatosos en un segmento de colon.

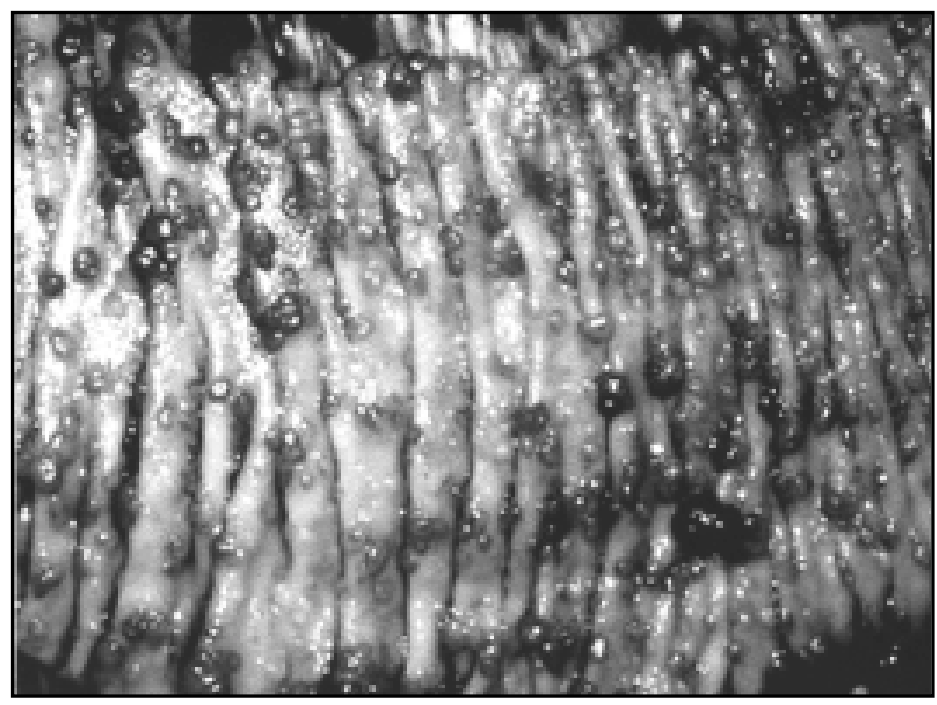

quimas, intestinales y extraintestinales ${ }^{3,11-15}$. La transformación aparentemente inevitable de adenoma a adenocarcinoma colorrectal en los casos no tratados, y la erradicación de este riesgo con la colectomía profiláctica, ha centrado el enfoque de esta patología en sus aspectos quirúrgicos ${ }^{5-7,15-25}$. Sin embargo, y con el propósito de disminuir aún más las tasas de mortalidad asociada con esta patología, se torna relevante el trabajo de pesquisa en los familiares de los pacientes operados. Así, no sólo podríamos estimular a los familiares para estudiarse y eventualmente operarse, sino que también podríamos obtener información relevante acerca de características clínicas y epidemiológicas de esta enfermedad. El objetivo de este trabajo es analizar los resultados tanto del tratamiento quirúrgico de los pacientes operados por PAF, como de la pesquisa de la enfermedad entre sus familiares.

\section{PaCIENTES y MÉTOdos}

Se revisaron los registros clínicos de los pacientes operados por PAF entre enero de 1977 y agosto de 2002, y se diseñó una base de datos para la inclusión prospectiva de los futuros pacientes portadores de esta patología. Se estudiaron los aspectos clínicos, quirúrgicos y evolución postoperatoria. El seguimiento fue en base a controles clínicos, vía telefónica y certificados de defunción del Registro Civil. Por otra parte, se realizó una encuesta telefónica a los pacientes índices, con el fin de identificar los familiares portadores de la enfermedad y poder así diseñar el árbol genealógico de cada familia. En ellos, se investigó dirigidamente: edad, sexo, eventual estudio o diagnóstico de PAF, antecedente de colectomía, presencia de cáncer, fallecimiento y su causa. Se identificó también a los familiares que cumplían con los criterios de algún estudio de pesquisa familiar (tener un familiar de primer grado con PAF, ser mayor de 12 y menor de 50 años). Entre ellos, se investigó aquellos que efectivamente se habían realizado algún estudio.

\section{RESULTADOS}

En 25 años, en el departamento de Cirugía Digestiva de la Pontificia Universidad Católica de Chile, se operaron 15 pacientes: 6 mujeres y 9 hombres con PAF, con una edad mediana de 28 años (19-48). El 67\% de los pacientes consultó por síntomas: 9 por hematoquezia (60\%) y 1 por osteoma frontal (7\%). Seis pacientes (40\%) tenían manifestaciones cutáneas; 2 (13\%) hiperplasia congénita del epitelio retiniano (HCEPR) y 2 (13\%), pólipos gastroduodenales. Cinco pacientes consultaron en el contexto de un estudio de pesquisa familiar (33\%).

No se evidenciaron tumores desmoides ni cáncer en otros parénquimas durante la evaluación preoperatoria. En 4 pacientes (27\%) se realizó una proctocolectomía e ileostomía terminal, en $3(20 \%)$ una proctocolectomía con reservorio ileal y en 8 (53\%), 
una colectomía total con anastomosis ileo-rectal, la última de ellas por vía laparoscópica ${ }^{25}$. Así, $73 \%$ de las cirugías fueron con preservación del esfínter anal (Figura 2). El tiempo operatorio y estadía mediana fueron de $4 \mathrm{~h}(3-7,5)$ y 11 días (6-15), respectivamente. Hubo sólo una complicación postoperatoria secundaria a una filtración del muñón rectal, en un paciente sometido a una proctocolectomía e ileostomía terminal, que se resolvió con terapia antibiótica. No hubo mortalidad operatoria.

La histopatología confirmó más de 100 pólipos en todos los pacientes. Se constató cáncer en 3 pacientes, todos mayores de 40 años. Un paciente tenía enfermedad metastásica al momento de la cirugía, la cual fue de carácter paliativo. El seguimiento se realizó en 12 pacientes (80\%), con una mediana de 68 meses (3-300). Un paciente murió (8\%) a los 18 meses postcirugía por evolución de su enfermedad metastásica (descrito previamente). Otro falleció secundario a SIDA 10 años después, sin evidencias de cáncer. La sobrevida enfermedad específica alcanza 92\%.

Dos mujeres (16\%) fueron reintervenidas por desarrollo de tumores desmoides: uno en pared abdominal y otro en el mesenterio. No se desarrollaron tumores en otros parénquimas, ni tampoco en el muñón rectal de los pacientes con anastomosis íleo rectal (AIR), los que fueron seguidos con rectoscopia seriada cada 6 meses. En 75\% de ellos, la aparición de nuevos pólipos se controló con polipectomías y tratamiento con sulindaco o inhibidores de la Cox-2. Sólo en uno de ellos se completó la proctectomía con confección de un reservorio ileal.

Respecto a la función anal, la frecuencia defecatoria mediana de los pacientes con AIR y reservorio ileal era de 4 deposiciones diurnas. Ninguno refirió incontinencia o diarrea invalidante.

Se contactó telefónicamente a 10 de los 15 pacientes operados (67\%), estructurando el árbol genealógico de 6 familias. Se identificó un total de 122 familiares, con una edad mediana de 27 años (1-84). De ellos, 82 tenían indicación de estudio de pesquisa de PAF por su edad, y sólo 27 (33\%) se habían estudiado alguna vez con colonoscopia, lo que se refleja en una familia en que sólo 18\% de los familiares con indicación de estudio se había realizado un examen endoscópico (Figura 5). No se registró otro estudio de pesquisa (genético o fondo de ojo). Hasta el momento de completar la revisión de los antecedentes, del total de familiares identificados, habían 36 miembros vivos que cumplen criterios para la realización de exámenes de pesquisa de PAF y que no han sido estudiados. La edad mediana de estos familiares es de 24 años (12-50).

De los 122 familiares, 36 (29\%) tenían diagnóstico confirmado de PAF (por antecedentes de colectomía). En 22 de éstos (61\%) se encontró cáncer colorrectal durante la cirugía, y 20 de ellos (91\%) fallecieron por la progresión de su neoplasia (Figura 3), arrojando una tasa de mortalidad de los familiares operados de $56 \%$, a una edad mediana de 43 años (22-54). Esto se ejemplifica en una de las familias en que 8 de los 11 miembros con PAF habían fallecido por un cáncer colorrectal (Figura 4). De las seis familias identificadas, en dos (28\%) se sospechó una mutación de novo por no tener antecedentes familiares de PAF en la generaciones anteriores.

\section{Discusión}

La PAF consiste en un conjunto de alteraciones intestinales y extraintestinales producto de una mutación a nivel del gen APC, ubicado generalmente en el brazo largo del cromosoma 5 (Figura 6) ${ }^{2-10}$. La caracterización de este gen, en 1991, ha permitido precisar diferentes mutaciones y asociarlas a distintas variantes de la enfermedad ${ }^{5-7,15-25}$. La presencia de

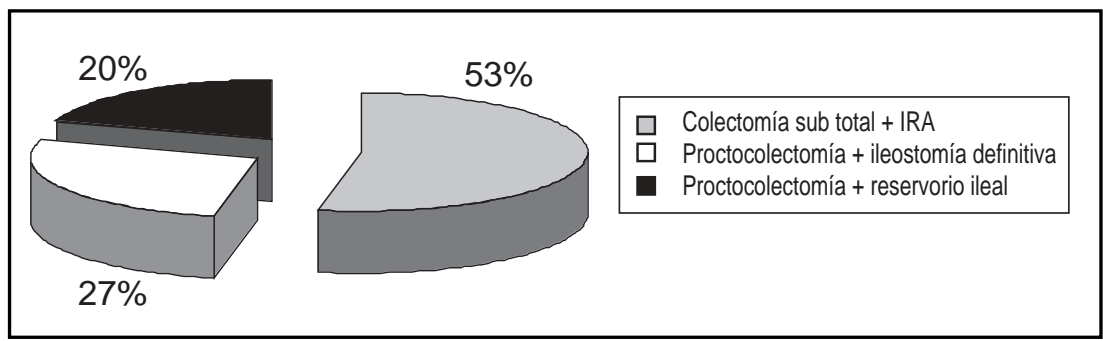

Figura 2. Distribución porcentual de los diferentes tipos de cirugía de los pacientes operados. 
Figura 3. Familiares de los pacientes operados por PAF. De los 36 familiares con PAF, 22 evolucionaron hacia un cáncer colorrectal. De éstos últimos, sólo dos están vivos.
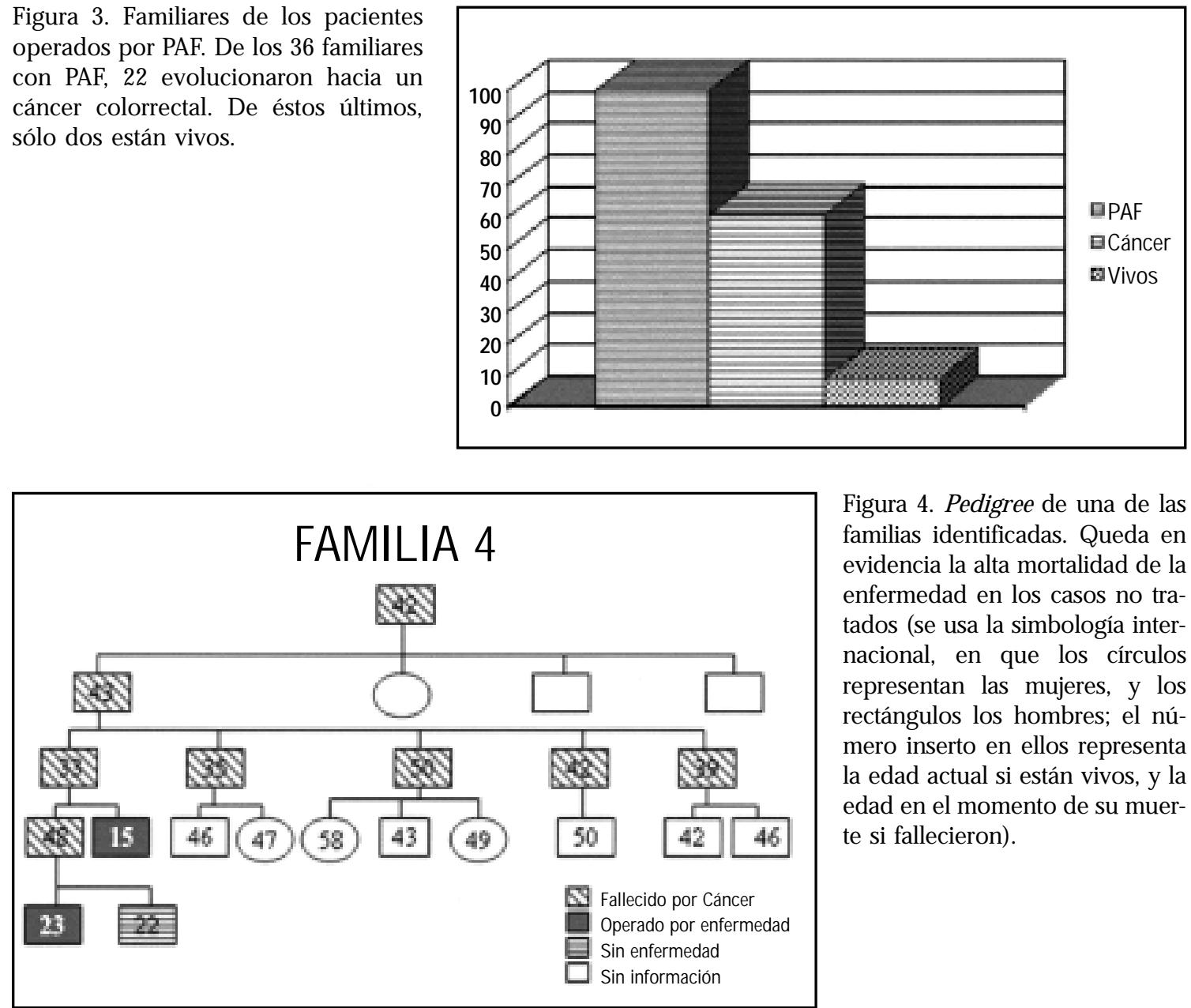

Figura 4. Pedigree de una de las familias identificadas. Queda en evidencia la alta mortalidad de la enfermedad en los casos no tratados (se usa la simbología internacional, en que los círculos representan las mujeres, y los rectángulos los hombres; el número inserto en ellos representa la edad actual si están vivos, y la edad en el momento de su muerte si fallecieron).

Figura 5. Pedigree de otra de las familias identificadas. Queda en evidencia la gran proporción de familiares no estudiados, a pesar de registrar en tres de sus parientes, varias muertes por cáncer colorrectal (se usa la simbología internacional, en que los círculos representan las mujeres, y los rectángulos los hombres; el número inserto en ellos representa la edad actual si están vivos, y la edad en el momento de su muerte si fallecieron).

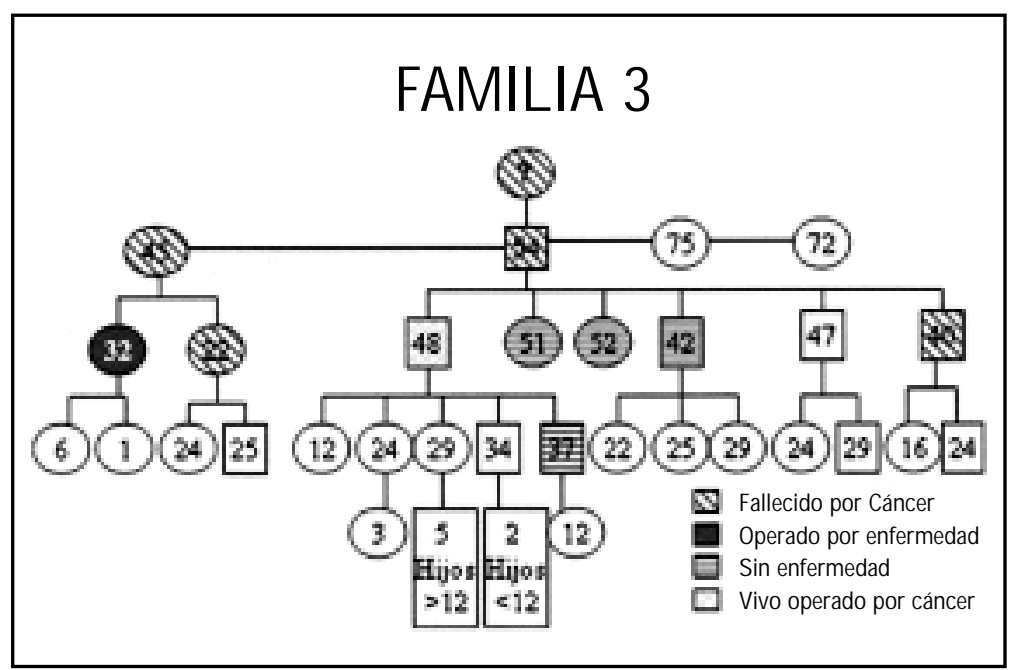


esta mutación en la línea germinal y su penetrancia cercana a 100\% hacen que la probabilidad de transmitirse a la descendencia sea de $50 \%{ }^{27}$. Si bien la mayoría de los casos son hereditarios, cerca de $30 \%$ de los casos se debe a una mutación de novo no presente en los padres del afectado 9,17,27. Los resultados de esta serie son comparables con esa proporción. Sin duda, la alteración más característica consiste en la poliposis colorrectal, que se define clásicamente por la presencia de más de 100 pólipos adenomatosos en ese segmento del tubo digestivo. La evolución aparentemente inevitable de alguno de estos pólipos hacia un adenocarcinoma constituye su peculiaridad más destacada ${ }^{5-7,15-25}$. La mortalidad de los casos no tratados sería, por lo mismo, inevitable. Por ello, esta enfermedad ofrece al cirujano una de las pocas oportunidades para prevenir el desarrollo de un cáncer colorrectal, mediante la colectomía profiláctica de los pacientes portadores de una PAF de reciente diagnóstico. La baja morbimortalidad asociada a las actuales altemativas de tratamiento quinúrgico de la enfermedad y sus buenos resultados a largo plazo, tanto respecto de la eventual aparición de un cáncer como de la funcionalidad esfinteriana, han definido a la cirugía como el tratamiento de elección para la PAF con o sin cáncer $7,18,19,24,28$.

Los resultados de nuestra serie apoyan la baja morbimortalidad de las diferentes opciones quirúrgicas, muestran la factibilidad de realizar cirugías que preserven el esfínter anal en la mayoría de los casos, y dejan al paciente con una frecuencia defecatoria aceptable que no influye en su calidad de vida. Con respecto a las distintas alternativas quirúrgicas, debemos destacar que ésta es una serie histórica que incluye pacientes operados antes del desarrollo del concepto del reservorio ileal (comienzos de la década 198089), por lo que los pacientes con expresión marcada de pólipos en el recto fueron sometidos a una proctocolectomía con ileostomía definitiva, determinando una secuela importante en pacientes asintomáticos y jóvenes. En la actualidad, esta cirugía se realiza en forma muy infrecuente en personas con cáncer del recto inferior o con alteración preoperatoria de la función del esfínter anal. De este modo, a la inmensa mayoría de los pacientes se les ofrece una colectomía total con anastomosis ileo-rectal o una proctocolectomía con reservorio ileal. El criterio de selección entre estas dos alternativas dependerá de la expresión fenotípica en el recto: con menos de 20 pólipos optamos por la anastomosis ileo-rectal y, en la actualidad, pensamos que la mejor opción es realizarla por vía laparoscópica ${ }^{25}$. El muñón rectal de estos pacientes puede controlarse con rectoscopias seriadas, y la aparición de nuevos pólipos puede prevenirse en gran medida con los inhibidores de la Cox-2 o con sulindaco. Así como la posibilidad de evitar una ileostomía definitiva es fuertemente valorada por los pacientes operados, pensamos que esta eventualidad debiera también influir en los familiares a la hora de decidir estudiar su eventual calidad de portador de PAF.

El pronóstico ominoso de los pacientes no tratados asociado a los buenos resultados de la colectomía profiláctica, ha promovido no sólo la resección quirúrgica en los pacientes ya diagnosticados, sino que también ha estimulado el desarmollo de programas de pesquisa en los familiares de los pacientes operados $3,8,15,17,27,29-31$. En países desarrollados se han creado registros regionales y nacionales para el estudio y la pesquisa precoz de los portadores de $\mathrm{PAF}^{28,32}$. En nuestro país no disponemos de dichos registros y los datos de pesquisa familiar de nuestra serie indican una importante desinformación por parte de los familiares y pacientes. Esto queda en evidencia al evaluar la baja tasa

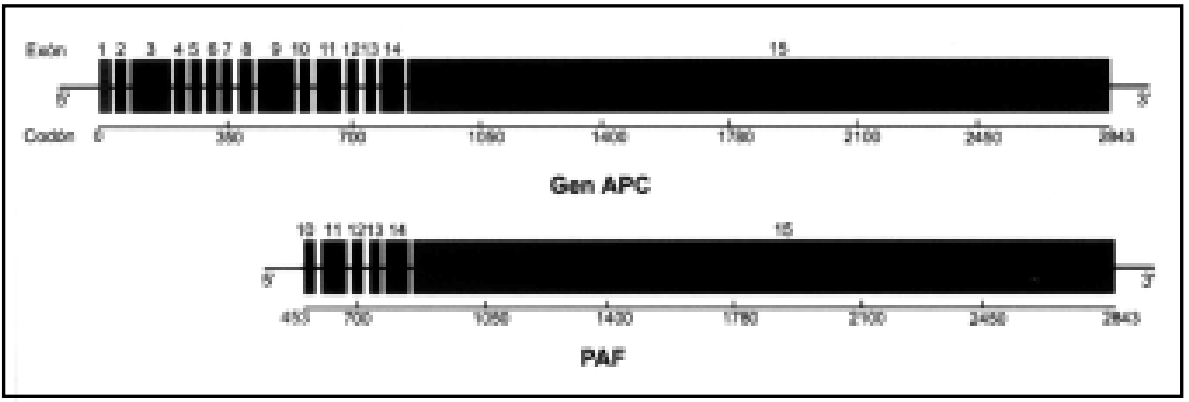

Figura 6. Gen APC y ubicación de la mutación de la variante clásica de la Poliposis Adenomatosa Familiar. 
de pacientes operados que consultaron en el marco de un estudio de pesquisa familiar: la mayoría lo hizo por tener ya algún síntoma. Esto mismo se desprende de la baja proporción de familiares que se realizó algún estudio de pesquisa familiar y de la alta tasa de familiares con cáncer al momento del diagnóstico de la PAF. La falta de información acerca de esta patología se refleja inevitablemente en la alta tasa de mortalidad de los pacientes con PAF de nuestra serie. A pesar de la baja incidencia de la enfermedad, nos parece indispensable y urgente el desarrollo de programas de pesquisa familiar que permitan estimular la colectomía profiláctica, y mejorar el ominoso pronóstico.

La pesquisa familiar ha demostrado ser efectiva en disminuir la edad y la incidencia de cáncer al momento del diagnóstico ${ }^{3}$. Diferentes centros han delineado recomendaciones para el estudio de los familiares en riesgo de desarrollar una $\mathrm{PAF}^{28,32}$. Están dirigidas a familiares de primer grado, planteando el inicio del estudio a los 10-12 años y mantenerlo en forma periódica hasta los 50 años; por la bajísima posibilidad de iniciar una PAF a esta edad (riesgo de 1:1.000), se recomienda seguir las normas estándar de pesquisa de cáncer colorrectal del grupo etario correspondiente ${ }^{17,27}$. El estudio de los familiares puede realizarse con colonoscopias seriadas, o mediante estudios genéticos. En nuestro país, el estudio de pesquisa familiar se ha realizado clásicamente mediante la endoscopia baja. Nuestra serie no evidenció ningún familiar o paciente estudiado con otro método. La colonoscopia debiera realizarse cada uno o dos años; desafortunadamente, es un estudio invasivo, con una receptividad mala por parte de los pacientes y probablemente difícil de realizar con una adherencia satisfactoria.

Desde la identificación y caracterización de las mutaciones responsables de la PAF, se han desarmollado diferentes estudios genéticos con el fin de identificar la mutación específica en el caso índice, y luego investigar su presencia en los familiares en riesgo. Entre los estudios más utilizados, por su facilidad y menor costo, así como por su alta sensibilidad, destaca el test de la proteína truncada (TPT). Consiste en la amplificación de segmentos específicos del gen APC del paciente portador de PAF, mediante PCR o (RT)-PCR que se transcriben y traducen in vitro; los productos pépticos originados a partir de estos diferentes segmentos del gen APC se analizan en un gel de electroforesis. Si el segmento amplificado posee una mutación, originará en $96 \%$ de los casos una proteína truncada» (es decir de menor tamaño) y este menor tamaño provocará una migración más rápida y una banda diferenciada en el gel (Figura 7). Se identifica el segmento de ADN que produjo esta proteína truncada, y se secuencia de tal forma de identificar los nucleótidos mutados. El uso del TPT permite identificar la mutación en cerca de 80\% de los pacientes con PAF.

$\mathrm{Si}$ el TPT resulta negativo (no se identifica proteína truncada), el paso siguiente es secuenciar la totalidad del ADN del gen APC. Desgraciadamente, este es un método consumidor de tiempo y de recursos, por lo que debe reservarse sólo para estos casos en que no se pudo determinar alguna alteración genética.

Una vez identificada la mutación del caso índice, se puede estudiar a los familiares de la misma forma. La detección de la mutación en un familiar (estudio mutacional positivo), debe proseguir con el estudio endoscópico, y planificar la colectomía profiláctica en

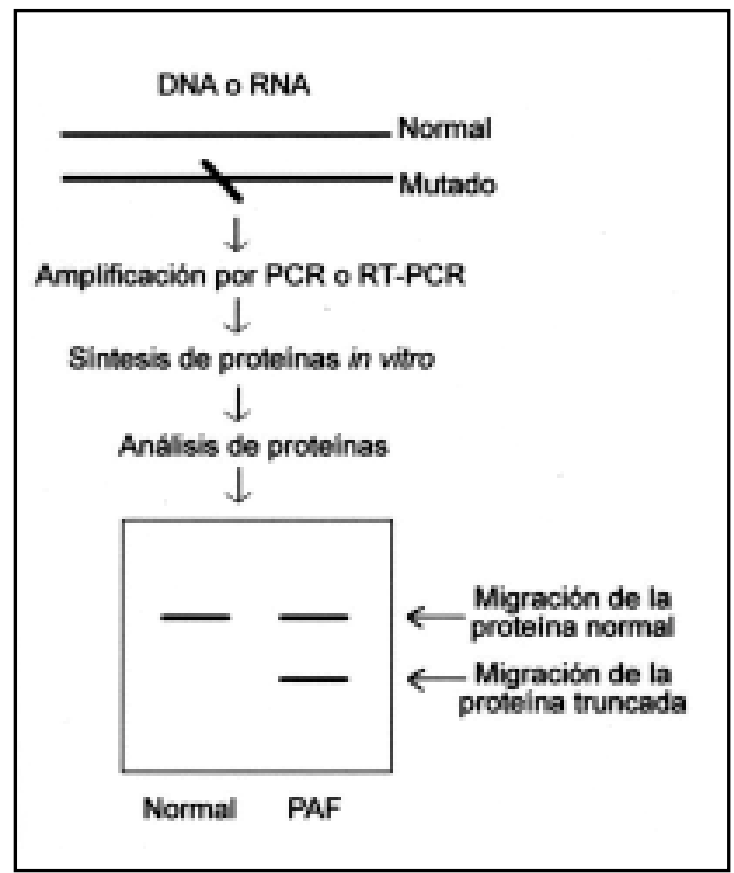

Figura 7. Test de la proteína truncada. Si el segmento amplificado posee una mutación, originará una proteína truncada, y su menor tamaño provocará una migración más rápida y una banda diferenciada en el gel de electroforesis. 
acuerdo con el paciente. Si luego de identificar la mutación en el caso índice, ésta no se encuentra en el familiar estudiado (estudio mutacional negativo), éste no seńa portador de la mutación y tendría, por tanto, el mismo riesgo de desamollar un cáncer colomectal que la población general, por lo que debiera acogerse a las normas de pesquisa de CC de la población general. En algunos casos sucede que, a pesar de secuenciar la totalidad del ADN del gen APC de un paciente con PAF, no se encuentra la mutación. En estos casos, la pesquisa familiar debe realizarse con colonoscopia según las normas clásicas ${ }^{16,26}$.

El estudio genético de los familiares posee varias ventajas. Es un examen no invasivo (linfocitos obtenidos a partir de una muestra de sangre) $\mathrm{y}$, por lo tanto, con una aceptación claramente mejor que la colonoscopia, lo que mejoraría en forma importante las bajas tasas de estudio de pesquisa que muestra nuestra serie. $\mathrm{Si}$ el estudio mutacional de estos familiares es negativo, se descarta la eventualidad de una PAF, evitando así controles repetidos con colonoscopia; por otra parte, elimina el grado de incertidumbre y angustia tanto para estos familiares como para su descendencia. En los casos con estudio mutacional positivo, permite diagnosticar la enfermedad y planificar la cirugía en forma programada e idealmente antes de la aparición de un CC.

Sus desventajas radican esencialmente en su elevado costo y en los casos en que no se logra identificar la mutación en el caso índice (mutación no detectada), los que deben continuar en forma tradicional con colonoscopias. Además, los familiares permanecen con la incertidumbre acerca de su calidad de portador de PAF. En esta situación, es fundamental explicar a los familiares la inconveniencia de realizarse estudios mutacionales, ya que la no detección de la mutación no indica que no sean portadores de una PAF, sino simplemente que el examen no es capaz de detectarla. Por ello, la interpretación de los resultados de un estudio genético debe realizarse con la máxima cautela, por las implicancias que tienen en el estudio y pronóstico del paciente.

\section{REFERENCIAS}

1. Fulerton D, López KF, Rahmer A. Cáncer colorrectal hereditario no poliposo: tratamiento quirúrgico y análisis de genealogías. Rev Méd
Las bajas tasas de realización de estudios de pesquisa familiar, la alta prevalencia de cáncer en los pacientes operados por PAF, la elevada mortalidad de estos pacientes y la temprana edad de los pacientes vivos en riesgo de portar una PAF que evidenciamos en esta serie sugieren la importancia de iniciar programas de detección precoz de esta enfermedad. Nos parece que las ventajas de los estudios mutacionales, especialmente en cuanto a sensibilidad y especificidad, como respecto de su mínima invasividad, debieran permitir que estos estudios sean incorporados gradualmente, si queremos mejorar el pronóstico de esta patología. Por otra parte, y probablemente como una etapa previa, debiera iniciarse un programa multicéntrico de registro de las familias con PAF a través del territorio nacional. Parece urgente planificar terapias de información a los pacientes y familiares, así como unificar la información disponible en los diferentes centros con el fin de disponer de una base de datos que nos permita extraer información real acerca de la magnitud de esta enfermedad en nuestro país.

En conclusión, la PAF es una enfermedad de elevada mortalidad sin tratamiento. El manejo quirúrgico presenta una baja tasa de morbimortalidad, permite mantener la continuidad del tránsito intestinal en la mayońa de los casos, ofrece al paciente una frecuencia defecatoria aceptable y reduce el riesgo de cáncer colomectal si se realiza en forma precoz. La tasa de estudios de pesquisa entre los familiares es baja, con una tasa de cáncer colomectal y mortalidad entre los portadores de PAF elevada. Se requiere desamollar exámenes de pesquisa para familiares de pacientes con PAF que permitan diagnosticar la enfermedad precozmente. Nos parece que la especificidad y no invasividad de los estudios genéticos debieran permitir mejorar las tasas de pesquisa familiar precoz, favorecer la colectomía profiláctica previa a la aparición de un CC, y disminuir las altas tasas de mortalidad que sugiere esta serie. Los costos de estos estudios son su mayor inconveniente, y su interpretación debe realizarse con máxima cautela. Asimismo, se sugiere la necesidad de crear un registro nacional de PAF.

Chile 2004; 132: 539-47.

2. Azolas C, Bocic G, Jensen C, Garrido R, Pérez G. Poliposis familiar: estudio familiar. Rev Chir Cir 1994; 46: 418-22.

3. Campbell W, Spence R, Parks T. Familial adenoma- 
tous polyposis. Br J Surg 1994; 81: 1722-3.

4. Chung D. The genetic basis of colorectal cancer: insights into critical pathways of tumorigenesis. Gastroenterology 2000; 119: 854-65.

5. Cole T, Sleightholme H. ABC of colorectal cancer. The role of clinical genetics in management. BMJ 2000; 321: 943-6.

6. Hernegger G, Moore H, Guilem J. Attenuated familial adenomatous polyposis: an evolving and poonly understood entity. Dis Colon Rectum 2002; 45: 127-36.

7. Jang Y, Steinhagen R, Heimann T. Colorectal cancer in familial adenomatous polyposis. Dis Colon Rectum 1997; 40: 312-6.

8. Petersen G, Brensinger J, Johnson K, Giardielo F. Genetic testing and counseling for hereditary forms of colorectal cancer. Cancer 1999; 86: 2540-50.

9. Rustin R, Jagelman D, McGannon E, Fazio V, Lavery I, WEAKLEY F. Spontaneous mutation in familial adenomatous polyposis. Dis Colon Rectum 1990; 33: 52-5.

10. YANG V. APC as a checkpoint gene: the beginning or the end? Gastroenterology 2002; 123: 935-9.

11. Kropilak M, Jagelman D, Fazio V, Lavery I, McGanNON E. Brain tumors in familial adenomatous polyposis. Dis Colon Rectum 1989; 32: 778-82.

12. Norton I, Gostout C. Management of periampullary adenoma. Dig Dis 1998; 16: 266-73.

13. Piace R, Simmang C. Hyperplastic-adenomatous polyposis syndrome. J Am Coll Surg 1999; 188: 503-7.

14. Chapman P, Church W, Burn J, Gunn A. Congenital hypertrophy of retinal pigment epithelium: a sign of familial adenomatous polyposis. BMJ 1989; 298: 353-4.

15. Church J, McGannon E, Burke C, Clark B. Teenagers with familial adenomatous polyposis: what is their risk for colorectal cancer? Dis Colon Rectum 2002; 45: 887-9.

16. Giardielo F, Brensinger J, Petersen G. AGA technical review on hereditary colorectal cancer and genetic testing. Gastroenterology 2001; 121: 198-213.

17. Wong N, Lasko D, Rabelo R, Pinsky L, Gordon P, FOULKES W. Genetic counseling and interpretation of genetic tests in familial adenomatous polyposis and hereditary nonpolyposis colorectal cancer. Dis Colon Rectum 2001; 44: 271-9.

18. Bjork J, Akerbrant H, Iseuus L, Svenberg T, Oresiand T, PAHLMAN L ET AL. Outcome of primary and secondary ileal pouch-anal anastomosis and ileorectal anastomosis in patients with familial adenomatous polyposis. Dis Colon Rectum 2001; 44: 984-92.
19. Church J, Fazio V, Lavery I, OAKiey J, Milsom J, McGanNon E. Quality of life after prophylactic colectomy and ileorectal anastomosis in patients with familial adenomatous polyposis. Dis Colon Rectum 1996; 39: 1404-8.

20. Kartheuser A, Parc R, Penna C, Tiret E, Frileux P, HanNoun L ET AL. Ileal pouch-anal anastomosis as the first choice operation in patients with familial adenomatous polyposis: a ten-year experience. Surgery 1996; 119: 615-23.

21. Nyam D, Briliant P, Dozois R, Kelly K, Pemberton J, WoLfF B. Ileal pouch-anal canal anastomosis for familial adenomatous polyposis: early and late results. Ann Surg 1997; 226: 514-9.

22. Sagar P, Pemberton J. Operations for familial adenomatous polyposis. Surg Oncol Clin NAm 1996; 5: 675-89.

23. Setti-CarRaro P, Nichows R. Choice of prophylactic surgery for the large bowel component of familial adenomatous polyposis. Br J Surg 1996; 83: 885-92.

24. van Duijvendijk P, Slors J, TaAt C, Oosterveid P, Vasen H. Functional outcome after colectomy and ileorectal anastomosis compared with proctocolectomy and ileal pouch-anal anastomosis in familial adenomatous polyposis. Ann Surg 1999; 230: 648-54.

25. Ziv Y, Church J, OAKiey J, McGannon E, Schroeder T, FAzIO V. Results after restorative proctocolectomy and ileal pouch-anal anastomosis in patients with familial adenomatous polyposis and coexisting colorectal cancer. Br J Surg 1996; 83: 1578-80.

26. López KF, Soto DG, Pinedo MG, Martínez C, Saenz R. Colectomía total por vía laparoscópica en Poliposis Adenomatosa Familiar. Rev Chil Cir 2003; 55: 385-8.

27. Rabelo R, Foulkes W, Gordon P, Wong N, Yuan Z, MacNamara E ET aL. Role of molecular diagnostic testing in familial adenomatous polyposis and hereditary nonpolyposis colorectal cancer families. Dis Colon Rectum 2001; 44: 437-46.

28. Moertel C, HiL J, Adson M. Surgical management of multiple polyposis. Arch Surg 1970; 160: 521-6.

29. JANNe P, MAYeR R. Chemoprevention of colorectal cancer. N Engl J Med 2000; 342: 1960-8.

30. KRONBORG O. Screening for early colorectal cancer. World J Surg 2000; 24: 1069-74.

31. RHodes M, Bradburn D. Overview of screening and management of familial adenomatous polyposis. Gut 1992; 33: 125-31.

32. Bussey H, Eyers A, Ritchie S, Thomson J. The rectum in adenomatous polyposis: the St Mark's policy. Br J Surg 1985; 72: 29-31. 